\title{
Probing the cellular size distribution in cell samples undergoing cell death
}

\author{
Emilie Franceschini ${ }^{\mathrm{a}, *}$, Laure Balasse ${ }^{\mathrm{b}}$, Sandrine Roffino ${ }^{\mathrm{c}, * *}$, Benjamin \\ Guillet $^{\mathrm{b}}$ \\ ${ }^{a}$ Aix-Marseille Université, CNRS, Centrale Marseille, LMA, Marseille, France \\ ${ }^{b}$ Aix-Marseille Université, INSERM, INRA, C2VN, Marseille, France \\ ${ }^{c}$ Aix-Marseille Université, CNRS, ISM, Marseille, France
}

\begin{abstract}
A polydisperse scattering model adapted for concentrated medium, namely the polydisperse structure factor model, was examined in order to explain the backscatter coefficients (BSCs) measured from packed cell samples undergoing cell death. Cell samples were scanned using high-frequency ultrasound in the 10-42 MHz bandwidth. A parameter estimation procedure was proposed in order to estimate the volume fraction and the relative impedance contrast that could explain the changes in BSC pattern by considering the actual change in cellular size distribution. Quantitative ultrasound parameters were estimated and related to the percentage of dead cells determined by flow cytometry. The standard deviation of scatterer size distribution extracted from the polydisperse structure factor model and the spectral intercept were found to be strongly correlated to the percentage of dead cells $\left(r^{2}=0.79\right.$ and $r^{2}=0.72$, respectively). The current study contributes to the

${ }^{*}$ Corresponding Author: Dr. Franceschini Emilie, Aix-Marseille Université, CNRS, Centrale Marseille, LMA, Marseille, France ; Email, franceschini@lma.cnrs-mrs.fr; Phone, +33484524286

**Also at: Université Côte d'Azur, Nice, France
\end{abstract}


understanding of ultrasonic scattering from cells undergoing cell death towards the monitoring of cancer therapy.

Key words:

Quantitative ultrasound, Cell death, Polydispersity, Structure factor model, Scatterer size distribution 


\section{Introduction}

Quantitative UltraSound (QUS) techniques for determining tissue microstructure are promising tools to detect and quantify cell death, and thus monitor tumor response to therapy. The radiofrequency (RF) backscattered signals provide information about the tissue microstructure which is not resolvable by conventional ultrasound B-mode images. QUS techniques examine the frequency dependence of the signals backscattered from tissues and are used in a wide range of applications to differentiate normal versus diseased tissue and characterize tumors in the prostate (Feleppa et al. 1997), breast (Oelze et al. 2004), lymph node (Mamou et al. 2010) and thyroid (Lavarello et al. 2013). Spectral-based QUS parameters such as the spectral slope, the spectral 0-MHz intercept and the midband fit are derived from linear regression analysis of RF power spectra. Model-based QUS parameters, such as the average scatterer diameter (ASD) and acoustic concentration (AAC), can be obtained by fitting an ultrasonic scattering model to the measured backscatter coefficient (BSC). QUS techniques for monitoring cell death were first conducted on cell pellet biophantoms exposed to chemotherapeutics (Czarnota et al. 1997, Kolios et al. 2002, Brand et al. 2008, Brand et al. 2009). Cell pellet biophantoms consist of centrifuged cells mimicking densely packed cells in tumors and serve as highly simplified in vitro versions of real tumors. These in vitro studies demonstrated that apoptosis causes an increase in backscatter intensity and a change in QUS parameters (i.e., spectral slope, intercept, midband fit, ASD and/or AAC) (Kolios et al. 2002,

Brand et al. 2008, Brand et al. 2009). High frequency QUS (>20 MHz) has also been applied to in vivo animal models exposed to cancer radiotherapy or 
chemotherapy in order to differentiate between responding and non responding regions within tumors (Vlad et al. 2008, Tadayyon et al. 2015). Recent clinical applications have also demonstrated that low frequency QUS $(<10$ $\mathrm{MHz}$ ) is a power tool to predict breast tumor response to therapy (Sannachi et al. 2015, Tadayyon et al. 2017), even though lower frequencies cannot be used to provide an actual description of the cellular structures.

In order to develop QUS techniques and interpret QUS parameters for cancer therapy assessment, it is essential to understand which are the specific changes (in cell morphology and/or in cellular mechanical properties) during cell death that cause these changes in QUS parameters. The aforementioned QUS studies are generally based on classical ultrasound scattering models (i.e., spherical Gaussian model or fluid sphere model) which assume randomly and independently distributed scatterers. Under this assumption, the power of the backscattered signals increases linearly with the scatterer volume fraction and this linear relationship has been used to monitor the ASD and AAC. More recently, the concentrated ultrasound scattering model, namely the polydisperse structure factor model (SFM), has been proposed to explain the measured BSCs (or structure functions) from densely packed media (Han et al. 2015, Franceschini et al. 2014, 2016). The polydisperse SFM describes tissue as an ensemble of discrete scatterers and considers interference effects caused by the correlations among scatterer positions (coherent scattering) using a structure factor. Experiments on in vitro cell pellet biophantoms and ex vivo mouse tumor models demonstrated that the SFM is the most appropriate model to use for modeling densely packed cellular contents in tumors (Han et al. 2015, Franceschini et al. 2014, 2016, Muleki-Seya et al. 
2016). The SFM has also been used in a two-dimensional (2D) numerical study to explain the contribution of changes in cellular size variance to the increase in the BSC during a cell death process (Vlad et al. 2010). However, this 2D model-based approach cannot be applied to quantitatively comparing the 2D simulated BSCs and the experimentally measured BSCs.

The first objective of this work was to go further in the understanding of the BSCs measured from cells undergoing apoptosis. Ultrasonic backscatter measurements were performed at frequencies ranging from 10 to $42 \mathrm{MHz}$ on colon adenocarcinoma (HT29) cell samples treated with staurosporine, an inducer of apoptotic cell death. The polydisperse SFM was examined in order to explain the BSC measured from HT29 cells undergoing cell death. To this end, a parameter estimation procedure was proposed in order to estimate the volume fraction and the relative impedance contrast that could explain the change in BSC pattern by considering the actual change in cellular size distribution during cell death.

The second objective of this work was to blindly estimate the cellular size distribution in cell samples undergoing cell death. For this purpose, a novel approach was proposed, which consisted in fitting the polydisperse SFM to two BSCs measured before and after therapy. This novel approach makes it possible to estimate four QUS parameters: the mean scatterer radius, the standard deviation of the scatterer size distribution, the total volume fraction and the relative impedance difference. QUS parameters estimated by the polydisperse SFM were compared with the ASD and AAC estimated by the classical sparse scattering model, namely the fluid sphere model. Finally, the relationship between the percentage of dead cells and QUS parameters was 
investigated.

\section{Background: ultrasonic scattering theory}

This section briefly describes three ultrasonic scattering models: the polydisperse SFM, the monodisperse SFM and the fluid-sphere model. These theoretical models describe tissue as an ensemble of discrete scatterers and are based on several approximations for soft tissue scattering (Insana et al. 1990): far-field regime, Born approximation, no multiple scattering and incident plane wave propagation. Moreover, the scatterers are assumed to be non-overlapping spheres with identical acoustic properties.

The polydisperse structure factor model (SFM)

When considering an ensemble of spheres differing only in size with radius $x$ and scattering amplitudes $\Phi(k, x)$, the BSC is given by (Griffith et al. 1987)

$$
\begin{aligned}
& B S C_{\mathrm{SFMp}}(k)=n \int_{0}^{\infty}|\Phi(k, x)|^{2} f(x) \mathrm{d} x+ \\
& n \int_{0}^{\infty} \int_{0}^{\infty} \Phi\left(k, x_{1}\right) \Phi\left(k, x_{2}\right) H_{12}(k) f\left(x_{1}\right) f\left(x_{2}\right) \mathrm{d} x_{1} \mathrm{~d} x_{2},
\end{aligned}
$$

where $k$ is the wavenumber, $n$ is the scatterer number density, $f$ is the probability density function (PDF) of the scatterer radii, and $H_{12}$ is the partial structure function. In the present study, we use the analytical expression of Eq. (1), which exists when the scattering amplitude is derived from the fluid sphere form factor (Han \& O'Brien 2015)

$$
\Phi(k, x)=\frac{\gamma_{z}}{4 k}[\sin (2 k x)-2 k x \cos (2 k x)],
$$

where $\gamma_{z}$ is the relative acoustic impedance difference between the scatterer and the surrounding medium, and when the scatterer size distribution follows 
a gamma PDF (Griffith et al. 1987)

$$
f_{(a, \zeta)}(x)=\frac{1}{\zeta !}\left(\frac{\zeta+1}{a}\right)^{\zeta+1} x^{\zeta} e^{-(\zeta+1) x / a}
$$

\section{The fluid sphere model}

The fluid sphere model assumes a sparse distribution of spheres, such that the fluid spheres are considered to be randomly and independently distributed. In this peculiar case, the structure factor is equal to unity. Considering an ensemble of identical fluid spheres of radius $a$, the theoretical BSC 
using the fluid sphere model is given by (Insana et al. 1990)

$$
B S C_{\mathrm{FSM}}(k)=\frac{4}{9} n \gamma_{z}^{2} k^{4} a^{6} F F(k, a),
$$

where $n \gamma_{z}^{2}$ is the acoustic concentration (AAC) and $d(=2 a)$ is the scatterer diameter (ASD).

\section{Materials and Methods}

\section{Cell sample preparation}

Experiments were conducted with HT29 cell samples treated with staurosporine, a drug which induces mainly cell apoptosis (Qiao et al. 1996). Cells were grown at $37^{\circ} \mathrm{C}$ in T175 flasks filled with Dulbecco's modified Eagle's medium containing $4.5 \mathrm{~g}$ of glucose/liter and supplemented with $10 \%$ fetal calf serum. The cells were treated in culture with staurosporine in T175 flasks at 60-70\% confluence and then prepared as packed cell samples (i.e., cell pellets). The detailed protocol is described below. For forming one packed cell sample, around $2 \times 10^{8}$ HT29 cells are needed (corresponding approximately to three T175 flasks of non-treated cells or four T175 flasks of treated cells). The supernatant (containing dead cells) was removed or reserved from the T175 flasks, and cells were detached with accutase and washed in phosphate-buffered saline (PBS). Cells from flasks were collected and the supernatant, if reserved, was added to the collected cells. After homogenization with a pipette tip, $100 \mu \mathrm{L}$ of this cell suspension was withdrawn for flow cytometry analysis and $50 \mu \mathrm{L}$ for cell size analysis. The remaining suspension was centrifuged for 5 minutes at $1200 \mathrm{~g}$, then the medium was aspirated and $500 \mu \mathrm{L}$ of PBS +/+ was added. After homogenization with a 
pipette tip, the cells were transferred in an 8-well Nunc Lab-Tek II Chamber Slide System (Dominique Dutscher, Brumath, France) and was finally centrifuged for 5 minutes at $1700 \mathrm{~g}$ to form densely-packed cell samples. Packed cell samples mimic the spatial distribution and packing of cells in tumors (Vlad et al. 2010). The chamber was then placed in a plastic dish and immersed in PBS to allow ultrasound measurement.

A total of 24 cell pellet biophantoms were studied and divided into four series of experiments, each series including five treated cell samples and one non-treated cell sample. All the experiments in one series were conducted on the dame day, so that the non-treated cell sample could be used as control. The dose effect or time effect of staurosporine was investigated as follows:

- Dose effect 1: HT29 cells were treated for 24 hours with different drug doses of $0,0.125,0.25,0.50,0.75$ and $1 \mu \mathrm{M}$ with addition of supernatant. Adding the supernatant during the preparation of the packed cell sample allows the percentage of dead cells to be increased.

- Dose effect 2: Same as dose effect 1.

- Dose effect 3: Same as dose effect 1, except that the supernatant was not added during the preparation of the packed cell sample.

- Time effect: HT29 cells were treated with $0.5 \mu \mathrm{M}$ of staurosporine for $0,6,12,24,36$ and 48 hours without addition of supernatant during the preparation of the packed cell sample.

After the ultrasound measurement, the cell samples were fixed in $10 \%$ buffered formalin for three days, dehydrated in a graded ethanol series, 
cleared in methylcyclohexane and embedded in methyl methacrylate resin. The resin-embedded samples were sectioned, and the sections stained with toluidine blue. The histological sections allow us to observe structural changes occurring in the dying cells and to verify that the cell spatial distribution was homogeneous. An example of histological images is presented in Fig. 1.

\section{Cell size and death analysis}

For each experimental condition, cell radii were measured with a Scepter TM 2.0 cell counter (Millipore, Molsheim, France). Figure (2a) shows typical examples of radius distribution for cells treated with $0.5 \mu \mathrm{M}$ of staurosporine for 0,12 and 24 hours. The cell size distribution was bimodal, with small cellular fragments (mean radius $a_{s} \approx 2.35 \mu \mathrm{m}$ ) and large cells (mean radius $\left.a_{l} \approx 6 \mu \mathrm{m}\right)$. The probability density function (PDF) of the cell radius $x$ was fitted with a mixture of two gamma PDFs $f$ with mixing parameter $p$ and was defined as:

$$
F_{\left(a_{s}, \zeta_{s}, a_{l}, \zeta_{l}\right)}(x)=p f_{\left(a_{s}, \zeta_{s}\right)}(x)+(1-p) f_{\left(a_{l}, \zeta_{l}\right)}(x)
$$

where $f_{\left(a_{i}, \zeta_{i}\right)}$ are the gamma PDF defined in Eq. (3) and the subscripts $s$ and $l$ are used for the smaller cellular fragments and for the larger cells, respectively.

Flow cytometry using Annexin V/7-AAD was performed to quantify cell death. For each condition, the $100 \mu \mathrm{L}$ withdrawn sample of cells was centrifuged at $500 \mathrm{~g}$ for $5 \mathrm{~min}$ and resuspended in $100 \mu \mathrm{L}$ of ice-cold Binding Buffer. The cell suspension was then incubated for 15 minutes on ice in the dark with $10 \mu \mathrm{L}$ of Annexin Vfluorescein isothiocyanate (FITC) prediluted 1:100 in Binding Buffer and $20 \mu \mathrm{L}$ of 7-AAD (Annexin V-FITC / 7-AAD 
kit, Beckman Coulter, Marseille, France). Then, $400 \mu \mathrm{L}$ of ice-cold Binding Buffer was added before quantification with Gallios flow cytometer (Beckman Coulter, France). Approximately 2500 events were measured for each condition. The flow cytometry analysis makes it possible to estimate the percentage of viable cells and dead cells (in early apoptosis, late apoptosis and necrosis).

\section{Ultrasound data acquisition and BSC measurements}

Ultrasonic data were acquired using a high frequency ultrasound system (Vevo 770, Visualsonics Inc, Toronto, Canada). Two probes, RMV 710 and RMV 703, were used in B-mode. For the RMV 710 and the RMV 703 probes, the oscillating single-element focused circular transducers had center frequencies of 20 and $30 \mathrm{MHz}$ with $-10 \mathrm{~dB}$ bandwidths of 10-32 and 18-42 $\mathrm{MHz}$, focuses of 15 and $10 \mathrm{~mm}$ and f-numbers of 2.1 and 2.5, respectively. Raw RF data were digitized at a sampling frequency of $250 \mathrm{MHz}$ with 12-bit precision using a high-speed acquisition card (CS12501, Gage, Lockport, IL, USA).

During the experiments, the focus of each transducer was positioned $1 \mathrm{~mm}$ below the PBS/cell pellet biophantom interface. A translation stage (Physik Instrument, model M-403.4PD, Karlsruhe, Germany) controlled the probe motion. Five independent B-mode images were constructed from acquired $\mathrm{RF}$ echoes by translating the probe every $300 \mu \mathrm{m}$. Examples of ultrasonic B-mode scans obtained with the $20-\mathrm{MHz}$ center frequency probe are given in Fig. 3. For around 100 independent RF lines at the center of each B-mode image, echoes were selected in the focal zone with a rectangular window of $0.75 \mathrm{~mm}$. The power spectra of the gated RF signals were then averaged to 
provide $P_{\text {meas }}$. This procedure was repeated for each probe and each packed cell sample.

The attenuation was calculated for each sample to allow for attenuation compensation during the BSC estimation. The attenuation measurement was performed with a focused transducer with center frequency of $22 \mathrm{MHz}$ with -10 dB bandwidth 11-34 MHz, focus of $26 \mathrm{~mm}$ and f-number of 4 . The transducer focus was positioned at the interface between the sample and the Plexiglass planar reflector. An insertion-loss broadband technique was used to calculate the attenuation (in neper per centimeter) by comparing the spectra of the echoes reflected by the well base surface with and without the sample being inserted in the echo paths (Chen et al.1997).

The measured $B S C_{\text {meas }}$ were computed from $P_{\text {meas }}$ using the reference phantom method (Yao et al. 1990). The experimental and processing methods were described previously in detail in section III.C of (Franceschini et al. 2014). This procedure yielded a $B S C_{\text {meas }}$ compensated for the attenuation loss for each probe in the same region-of-interest. The two $B S C_{\text {meas }}$ were then combined to yield a single $B S C_{\text {meas }}$ over the combined bandwidths of the two transducers (i.e., 10-42 MHz).

\section{Optimization procedure for understanding the scattering from dead cells}

The polydisperse structure factor model was first examined in order to explain the measured $B S C_{\text {meas }}$ from packed cell samples undergoing cell death for all the 24 studied samples. Our hypothesis is that the changes in the measured $B S C_{\text {meas }}$ during the cell death process is mainly due to the changes in cell size distribution. In order to confirm or contradict this hypothesis, it was assumed that: 
1. the main sources of scattering are the whole cells and the contribution of cellular fragments $\left(a_{s} \approx 2.35 \mu \mathrm{m}\right)$ to the backscattering is negligible,

2. the large cells are gamma distributed, and the radius $a_{l}$ and gamma width factor $\zeta_{l}$ are known a priori and given by the Scepter TM 2.0 cell counter,

3. the total volume fraction $\phi_{l}$ is similar for all the cell pellet biophantoms since all the samples were prepared under the same centrifugation force,

4. the impedance relative contrast $\gamma_{z}$ does not change during the cell death process .

The unknown parameters are the total volume fraction $\phi_{l}$ and the impedance relative contrast $\gamma_{z}$, which are determined by minimizing the cost function $\mathcal{F}$ defined as (Franceschini et al. 2016):

$$
\begin{aligned}
& \mathcal{F}\left(\phi_{l}^{*}, \gamma_{z}^{*}\right)=\sum_{i=1}^{24} \\
& \sum_{j}\left\|\frac{B S C_{\text {meas }}^{M i}\left(k_{j}\right)-B S C_{\mathrm{SFMp}}^{M i}\left(k_{j}, a_{l_{i}}, \zeta_{l_{i}}, \phi_{l}, \gamma_{z}\right)}{B S C_{\text {meas }}^{M i}\left(k_{j}\right)}\right\|^{2}
\end{aligned}
$$

which synthesizes the 24 measurements $M_{i=1 \ldots 24}$ from the 24 studied cell pellet biophantoms. The measured $B S C_{\text {meas }}^{M i}$ corresponds to the $B S C_{\text {meas }}$ averaged over the five measurements (corresponding to the five acquired B-mode images) for each cell pellet biophantom. A routine fminsearch in MATLAB (The Mathworks Inc., Natick, MA), i.e., a Nelder-Mead simplex method, was employed to minimize the cost function $\mathcal{F}$. Afterwards, the theoretical BSCs computed with the estimated parameters were compared with the measured $B S C_{\text {meas }}$. 


\section{Blind estimation of QUS parameters}

Our second aim was to blindly estimate the QUS parameters. Two approaches were compared: 1) by fitting one measured BSC with a straight line or with the classical fluid sphere model, or 2) by fitting two measured BSCs before and after therapy with the polydisperse SFM.

Estimation of QUS parameters by fitting one measured BSC. Using a straight line model, the spectral slope and the spectral intercept were calculated (Lizzi et al. 1986). The spectral slope is the linear slope of the BSC as a function of frequency on a log-log scale. The spectral intercept is the extrapolation of the BSC linear fit to zero frequency. The slope is related to the effective scatterer size and the intercept is determined by the effective scatterer size and acoustic concentration.

The $\mathrm{ASD}^{*}$ and $\mathrm{AAC}^{*}$ were estimated by fitting one measured $B S C_{\text {meas }}$ with the fluid sphere model (Insana et al. 1990). These parameters were obtained by minimizing the mean square relative error between $B S C_{\text {meas }}$ and $B S C_{\mathrm{FSM}}$ given in Eq. (5) (Franceschini et al. 2016)

$$
\mathcal{C}_{1}\left(\mathrm{ASD}^{*}, \mathrm{AAC}^{*}\right)=\sum_{j}\left\|\frac{B S C_{\text {meas }}\left(k_{j}\right)-B S C_{\mathrm{FSM}}\left(k_{j}\right)}{B S C_{\text {meas }}\left(k_{j}\right)}\right\|^{2} .
$$

Estimation of QUS parameters by fitting two measured BSCs before and after therapy. A novel approach was proposed to estimate QUS scattering properties from the polydisperse SFM. This model parameterizes the BSC with four parameters: the mean scatterer radius $a$, the gamma width factor $\zeta$, the total volume fraction $\phi$ and the relative acoustic impedance contrast $\gamma_{z}$. Because of the large number of unknown parameters, we propose an estimation procedure using two measured $B S C_{\text {meas }}$ from non-treated sam- 
ple (denoted $B S C_{\text {meas }}^{n t}$ ) and treated sample (denoted $B S C_{\text {meas }}^{t}$ ). To model backscattering from cells undergoing cell death, some simplifying assumptions were considered. First, the non-treated cells are modeled as an ensemble of identical fluid spheres, whereas the treated cells are modeled as an ensemble of fluid spheres following a gamma PDF. Secondly, it is assumed that the mean scatterer radius $a$ and the relative acoustic impedance difference $\gamma_{z}$ do not change during the cell death process, and that the total volume fractions of cells are approximately the same for non-treated and treated conditions. The unknown parameters are the gamma width factor $\zeta$ of the treated cells, the mean scatterer radius $a$, the total volume fraction $\phi$ and the relative impedance difference $\gamma_{z}$, which are determined by minimizing the cost function $\mathcal{C}$ :

$$
\begin{aligned}
& \mathcal{C}_{2}\left(a^{*}, \zeta^{*}, \phi^{*}, \gamma_{z}^{*}\right)= \\
& \sum_{j}\left\|\frac{B S C_{\text {meas }}^{\mathrm{nt}}\left(k_{j}\right)-B S C_{\mathrm{SFMm}}\left(k_{j}, a, \phi, \gamma_{z}\right)}{B S C_{\text {meas }}^{\text {nt }}\left(k_{j}\right)}\right\|^{2} \\
& +\sum_{j}\left\|\frac{B S C_{\text {meas }}^{\mathrm{t}}\left(k_{j}\right)-B S C_{\mathrm{SFMp}}\left(k_{j}, a, \zeta, \phi, \gamma_{z}\right)}{B S C_{\text {meas }}^{t}\left(k_{j}\right)}\right\|^{2} .
\end{aligned}
$$

The first term represents the fit of $B S C_{\text {meas }}^{n t}$ with the monodisperse structure factor model $B S C_{\mathrm{SFMm}}$ given by Eq. (4). The second term represents the fit of $B S C_{\text {meas }}^{t}$ with the polydisperse structure factor model $B S C_{\mathrm{SFMp}}$ given by Eq. (1). The routine fmincon was employed to minimize the cost function $\mathcal{C}_{2}$ with the constraint conditions that $0 \leq a \leq 100 \mu \mathrm{m}, 1 \leq \zeta \leq 100$ and $0 \leq \gamma_{z} \leq 0.30$. For the constraint condition on the parameter $\phi$, two cases were studied: no a priori information with $0 \leq \phi \leq 1$, or a constraint that assumes concentrated medium with $0.68 \leq \phi \leq 1$. This point will be discussed later. The constrained optimization routine fmincon in MATLAB (The Mathworks Inc., 
Natick, MA), i.e., the interior-point method, was used because unconstrained optimization techniques sometimes gave unrealistic values for the estimated $\gamma_{z}$ (up to values of 0.7 )

In the sequel of the paper, the standard deviation of the scatterer diameter distribution $\sigma_{D}^{*}=2 a^{*} / \sqrt{\zeta^{*}+1}$ will be reported instead of the gamma width factor $\zeta^{*}$. For comparison with the fluid sphere model, the acoustic concentration from the polydisperse SFM is computed as: $\mathrm{AAC}^{*}=\frac{\phi^{*} \gamma_{z}^{* 2}}{\frac{\left(\zeta^{*}+3\right)\left(\zeta^{*}+2\right)}{\left(\zeta^{*}+1\right)^{2}} \frac{4 \pi a^{* 3}}{3}}$ [see Eq. (12) in (Franceschini et al. 2016)].

\section{Results}

\section{Flow cytometry, histology and cell size distribution}

The flow cytometry analysis revealed a mixture of apoptotic and necrotic cells in treated cells. The percentage of necrotic cells ranged between $6.7 \%$ and $29.2 \%$, but no relationship with dose or time exposure was found. The percentage of apoptotic cells increased when dose or time exposure to staurosporine increased (from $5.8 \%$ at 0 h to $19.9 \%$ at $24 \mathrm{~h}, 25.7 \%$ at $36 \mathrm{~h}$ and 36.3 at $48 \mathrm{~h}$ for time effect experiment, or from $5.9 \%$ at $0 \mu \mathrm{M}$ to $33.0 \%$ at $0.25 \mu \mathrm{M}$, to $48.5 \%$ at $0.50 \mu \mathrm{M}$ and $62.0 \%$ at $1 \mu \mathrm{M}$ for dose effect 1$)$, except for dose effect 2. For the latter, the percentage of apoptotic cells in treated samples was found equal to $60.2 \%$ at $0.25 \mu \mathrm{M}$, to $42.6 \%$ at $0.50 \mu \mathrm{M}$ and $51.8 \%$ at $1 \mu \mathrm{M}$, with no relationship with dose effect. In the following, the percentage of dead cells corresponds to the sum of percentages of cells undergoing early apoptosis, late apoptosis and necrosis. 
The histological study showed that in treated cells the staining of the nuclear material is uniform (see Fig. 1a), whereas numerous cells treated with staurosporine present characteristics of apoptosis (nuclear condensation and fragmentation) and characteristics of necrosis (cell swelling) (see Fig. 1b). Histological observations also demonstrated that whole cells are densely packed in treated and non-treated cell samples. For each histological slice, cellular surface fractions were calculated as the ratio between the white color area (corresponding to the absence of cells) and the total area. The cellular surface fractions were found to be comprised between 0.91 and 0.98 for all the studied (non-treated and treated) packed cell samples. This result was expected since all the packed cell samples were prepared under the same centrifugation force. The cellular volume fraction within the cell pellet biophantoms is related to the cellular area fraction that can be observed on those histological images (Fig. 1). That is why we can reasonably assume that the total volume fractions of cells are similar in all the 24 cell pellet biophantoms.

The cellular radius distribution measured by cell counter was fitted with a mixture of two gamma PDFs using Eq. (6). Table 1 summarizes the mean radii and gamma width factors for small cellular fragments $\left(a_{s}, \zeta_{s}\right)$ and large cells $\left(a_{l}, \zeta_{l}\right)$, as well as the percentage of volume fraction occupied by large cells $\Phi_{l}$. For all series of experiments, the non-treated cells exhibit a quasiunimodal size distribution $\left(\Phi_{l} \geq 0.99\right)$, a mean cell radius around $6.5 \mu \mathrm{m}$ and a narrow size distribution $(\zeta \geq 42)$. After cell death, the percentage of volume fraction occupied by large cells $\Phi_{l}$ decreases, and the gamma width factor $\zeta$ decreases, especially for the dose effect 1 and the time effect. Considering 
all the 20 treated cell samples, the averaged radius of large cells is equal to $a_{l}=6.6 \pm 0.9 \mu \mathrm{m}$, which is close to the mean radius of non-treated cell samples.

Figure 2(b) gives examples of histograms of the cell volume distribution. Even when the counts of small cellular fragments (mean radius $a_{s} \approx 2.35$ $\mu \mathrm{m}$ ) are greater than the counts of large cells (mean radius $a_{l} \approx 6 \mu \mathrm{m}$ ), the percentage of volume fraction occupied by the large cells $\Phi_{l}$ is always greater than 0.74 (see Table 1 and Figure 2(b)). Since the backscattering cross-section is proportional to the square of the scatterer volume in the low frequency, the contribution of cellular fragments $\left(a_{s} \approx 2.35 \mu \mathrm{m}\right)$ to the backscattering can be considered negligible.

Understanding of scattering: the change in cell size distribution mainly explains the change in BSC pattern on dying HT29 cell samples

The volume fraction estimated by the optimization procedure using Eq. (7) was found equal to $\phi_{l}^{*}=0.73$ and agrees well with the expected maximum packing for hard spheres (around 0.74). The relative impedance contrast was found equal to $\gamma_{z}^{*}=0.25$. By taking the acoustic parameters of individual viable cells estimated by (Falou et al. 2010) $(c=1535 \mathrm{~m} / \mathrm{s}$ and $\rho=1.09)$ and the acoustic properties of the surrounding medium close to those of water $\left(z_{0}=1.49 \mathrm{MRayl}\right)$, the relative impedance of viable cells is expected to be approximately 0.13 . Therefore, the estimated $\gamma_{z}^{*}=0.25$ seems to be in a reasonable range of value, but maybe slightly overestimated.

Figure 4 shows some examples of $B S C_{\text {meas }}$ versus frequency curves averaged over the five measurements (corresponding to the five acquired B-mode images) and the corresponding theoretical $B S C_{\text {theo }}$ computed with the poly- 
disperse SFM. Also given in Fig. 4 are the goodness-of-fit statistics, $R^{2}$, as defined in Eq. (2) in Ref. (Oelze \& O'Brien 2006). The theoretical $B S C_{\text {theo }}$ was computed with the polydisperse SFM using the radius distribution of large cells measured by the cell counter (see $a_{l}$ and $\zeta_{l}$ given in Table 1) and the fitting parameters $\phi_{l}^{*}=0.73$ and $\gamma_{z}^{*}=0.25$ obtained from the optimization procedure. For 19 BSC measurements out of 24 (i.e., $79 \%$ of the BSC curves), the theoretical and experimental BSCs share a similar pattern with goodness-of-fit $R^{2}$ values higher than 0.8 , as it can also be observed in Fig. 4. However, large differences between theory and experiments can also occur. For example, the experiment with $0.75 \mu \mathrm{M}$ of the dose effect 1 shows a negative value of the goodness of fit $\left(R^{2}=-0.19\right)$ meaning that a mere horizontal line passing through the average value of the $B S C_{\text {meas }}$ would fit data better than does the polydisperse SFM (see Fig. 4(b)). Note that only one BSC measurement out of 24 shows a negative value of the goodness of fit. Otherwise, 4 BSC measurements over 24 show goodness-of-fit value comprised between 0.5 and 0.8 .

The $B S C_{\text {meas }}$ and $B S C_{\text {theo }}$ averaged over the $10-32 \mathrm{MHz}$ bandwidth (corresponding to the frequency bandwidth of the RMV710 probe) are compared in Fig. 5 for all the 24 cell pellet biophantoms. A good correlation $\left(r^{2}=0.78\right)$ is found between the averaged $B S C_{\text {meas }}$ and $B S C_{\text {theo }}$ (Fig. 5).

To conclude, our approach hypothesizes that there is no change in impedance contrast during cell death and that both treated and non-treated cell samples have similar volume fractions of cells, so that only a change in scatterer size distribution has been considered. Despite the use of these simplifying assumptions, the good match obtained between theoretical and experimental 
BSCs in the majority of cases (Figs. 4 and 5) suggests that the change in whole cell size distribution is the predominant factor to explain the change in the BSC pattern in the HT29 cell samples during the dying process. (A more detailed discussion on these simplifying assumptions will follow later.)

Blind estimation of QUS parameters using the classical approaches: the spectral intercept correlates with the percentage of HT29 dying cells

For each cell pellet biophantom, five $B S C_{\text {meas }}$ were measured (corresponding to the five acquired B-mode images), and for each measured $B S C_{\text {meas }}$, the four classical QUS parameters (ASD*, AAC*, spectral slope, spectral intercept) were estimated. When using the fluid sphere model, the ASD* increases and the $\mathrm{AAC}^{*}$ decreases as the percentage of dead cells increases. Examples of $\mathrm{ASD}^{*}$ and $\mathrm{AAC}^{*}$ values are given in Fig. 6(a) for the series of experiments dose effect 1. When using a straight line model, the spectral slope decreases and the spectral intercept increases as the percentage of dead cells increases (data not shown).

The percentage of dead cells was plotted against the classical QUS parameters for the 24 studied cell pellet biophantoms, as shown in Fig. 6(b) with the spectral intercept parameter. The classical QUS parameters yield good correlation with the percentage of HT29 dying cells: $r^{2}=0.71$ for ASD*, $r^{2}=0.61$ for $\mathrm{AAC}^{*}, r^{2}=0.67$ for the spectral slope and $r^{2}=0.73$ for the spectral intercept. The highest correlation coefficient of $r^{2}=0.73$ suggests that the spectral intercept may be a pertinent parameter to assess the cell death index in an HT29 tumor exposed to chemotherapy. 
Blind estimation of QUS parameters using the novel approach

When considering the QUS parameters (ASD* $\mathrm{AAC}^{*}$ and $\sigma_{D}^{*}$ ) estimated by the novel approach based on the polydisperse SFM with the constraint $0 \leq \phi \leq 1$, the standard deviation of the scatterer diameter distribution $\sigma_{D}^{*}$ was found to increase with the percentage of dead cells, providing a good correlation coefficient $r^{2}=0.79$ (see Fig. 6(c)). No correlation was found between the percentage of dead cells and the other QUS parameters estimated by the novel approach (ASD* or $\left.\mathrm{AAC}^{*}\right)$.

Table 2 gives examples of QUS parameters (ASD*, AAC* and $\sigma_{D}^{*}$ ) estimated by the novel approach for the time effect experiment. Also given in Table 2 are the expected parameters: the expected ASD and standard deviation $\sigma_{D}$ given by the cell counter, and the expected AAC calculated by considering $\phi_{l}^{*}=0.73$ and $\gamma_{z}^{*}=0.25$. The strong constraint $0.68 \leq \phi \leq 1$ allows us to obtain QUS parameters close to the expected parameters, whereas the constraint $0 \leq \phi \leq 1$ leads to large differences between the expected and estimated ASD and AAC (Table 2). As a consequence, the actual standard deviation of actual cell size distribution and those estimated by the polydisperse SFM are strongly correlated $\left(r^{2}=0.69\right)$ when using the constraint $0.68 \leq \phi \leq 1$, and are moderately correlated $\left(r^{2}=0.46\right)$ when using the constraint $0 \leq \phi \leq 1$ (data not shown).

\section{Discussion and Conclusion}

\section{Understanding scattering}

It is generally assumed that the scattering within cell pellet biophantom or tumor mainly arises from the nuclei or from the whole cells (Oelze et 
al. 2006; Taggart et al. 2007; Franceschini et al. 2014; Muleki-Seya et al. 2016). The nucleus and whole cell are tightly interconnected and their size and properties change simultaneously during cell death (Vlad et al. 2009). That is why we conducted a study to assess if only a change in nuclear size could explain the change in BSC magnitude, as explained below.

The optimization procedure proposed in Eq. (7) was slightly modified by considering the nuclei as the main sources of scattering. More precisely, the first two assumptions were re-formulated as follows: 1) the main sources of scattering are the nuclei, and 2) the nuclei are gamma distributed and the radius $a_{n}$ and gamma width factor $\zeta_{n}$ can be calculated from the measurements with Scepter cell counter by considering a nucleus-to-cell ratio $\left(a_{n} / a_{l}\right)$ equal to 0.72 . This novel optimization procedure makes it possible to estimate a volume fraction $\phi_{n}^{*}=0.69$ and a relative impedance contrast $\gamma_{z_{n}}^{*}=0.36$. However, the estimated volume fraction did not match the expected volume fraction of nuclei $\phi_{n} \approx \phi_{c}\left(a_{n} / a_{l}\right)^{3}=0.27$ (by considering a volume fraction of densely packed whole cells $\left.\phi_{c}=0.74\right)$. Moreover, the value of the modified cost function $\mathcal{F}^{\prime}$ was found to be larger when considering the nuclei as the sources of scattering (when compared with the original cost function $\mathcal{F}$ in Eq. (7)). Therefore, the whole cell seems to play a major role in the BSC curves for the HT29 cell samples treated with staurosporine, as observed previously in biophantoms of viable K562 and CHO cells (Franceschini et al. 2014).

At the sub-cellular scale, the change in acoustic properties during cell death is still not well understood. Previous acoustic microscopy experiments (at $375 \mathrm{MHz}$ or at $0.9-1 \mathrm{GHz}$ ) were performed to measure acoustic properties 
on single viable and apoptotic cells (Taggart et al. 2007, Strohm et al. 2010). It was found that the apparent attenuation increased by $61 \%$ after apoptosis, whereas the other acoustic properties (sound speed, acoustic impedance, density, bulk modulus) were similar (Strohm et al. 2010). However, this apparent attenuation increase is difficult to interpret because it can be attributed to an increase in the cell absorption or backscatter, or a change in density of cell interior (Taggart et al. 2007), or due to diffraction effects on the cellular radius of curvature in the transducer focal region (Weiss et al. 2007). Previous QUS studies $(<40 \mathrm{MHz})$ were also conducted on packed cell samples exposed to cancer therapy and suggest that the increase in backscatter intensity can be due to the increase in acoustic impedance during the increasing compaction of nuclear material (Kolios et al. 2002, Pasternak et al. 2016) The nuclear condensation and fragmentation as well as the change in cellular size variance were also suggested to provide changes in scatterer size, spacing and distribution that might cause increasing backscatter intensity (Hunt et al. 2002, Brand et al. 2008, Vlad et al. 2010).

Because of the difficulty of differentiating between the influences of the change in acoustic impedance and the change in scatterer size variance, it was assumed, in a first approximation, that there is no change in acoustic impedance during cell death. Despite this simple approximation, it is very interesting to observe a good match between theoretical and experimental BSCs in Figs. 4 and 5 for the majority of cases (i.e., 19 BSC measurements out of 24). This suggests that the change in size distribution of large cells mainly contributes to the change in BSC pattern in dying HT29 cell samples. For 6 BSC measurements out of 24, the BSC experimental measurements 
could not be fully explained by our approach (see Figs. 4(b) and 4(d)). Investigating the sources that could explain this mismatch is difficult. It can be due for example to a change in cellular size variance (as proposed in this study) combined with a change in acoustic impedance. One might also consider a more complex scattering process by considering both nuclei and whole cells responsible for the scattering since histological observations reveal that the size distribution changes in both the nucleus and the cell during the cell death process. However, not all changes observed on histological images will result in scattering changes.

\section{Blinded estimation of QUS parameters}

The spectral slope is linked to the effective scatterer size. When considering a medium with scatterers differing only in size, the spectral slope decreases with the increase in scatterer size variance. As an example, theoretical BSCs were calculated in the $10-42 \mathrm{MHz}$ bandwidth by using Eq. (1) for a mean scatterer radius of $7 \mu \mathrm{m}$ and various gamma width factors $\zeta=90$, 50, 30 and 10. The corresponding spectral slopes were found to be equal to $4.10,4.05,4.00$ and 3.8, respectively. In our experimental study, the decrease in the spectral slope with the increase in the percentage of HT29 dying cells is thus consistent with an increase in the cellular size variance. Similar observations linked to the cellular size variance were previously observed in $\mathrm{FaDu}$, Hep-2 and C666-1 cell samples exposed to radiotherapy (Vlad et al. 2008, Vlad et al. 2010). When using the fluid sphere model, the ASD* was also found to increase together with the percentage of dying HT29 cells, which is consistent with the decrease in the spectral slope. 
In the present study, a novel approach was proposed to estimate QUS parameters from the analysis of HT29 cell samples before and after treatment. Plotting the percentage of dead cells against the standard deviation of scatterer diameter distribution $\sigma_{D}^{*}$ estimated by the polydisperse SFM shows a good correlation coefficient $r^{2}=0.79$ (Fig. 6(c)). The blind estimation of the change in cellular size variance may thus be a complementary parameter to assess the cell death index since the changes in cellular size variance reflect actual structural changes occurring during cell death. However, further study should be conducted on in vivo tumors to confirm the added value of this novel QUS parameter. Indeed, the assumptions of small changes in mean scatterer radius and in cellular volume fraction that are valid for the HT29 cell pellet biophantoms may not be adequate for actual tumors. For example, the cellular volume fraction within actual tumors could vary during the cell dying process, due to cell swelling during necrosis and/or due to cell shrinkage and formation of apoptotic bodies during apoptosis. Moreover, tumors have more complex structures than cell pellet biophantoms. The blood microvessels, extracellular matrix and tumor heterogeneity (responding and non-responding regions, different forms of cell death) may play a role in tumor scattering, as shown by (Han et al. 2013). Future studies should focus on 1) comparing the changes in backscattering between tumors and cell samples of the same cell line under the same chemotherapy to go further in the understanding of scattering from tumor, and 2) comparing the standard QUS parameters with those estimated by the polydisperse SFM on preclinical tumors under therapy to help in interpreting the standard QUS parameters. 


\section{Acknowledgements}

${ }_{490}$ We gratefully thank Samantha Fernandez from the European Center for 491 Research in Medical Imaging (CERIMED) for technical support in the prepa492 ration of cell samples and flow cytometry analysis. This work was carried 493 out thanks to the support of the French Agence Nationale de la Recherche ${ }_{494}$ (ANR) under the A*MIDEX project (ANR-11-IDEX-0001-02) funded by the 495 Investissements d'Avenir French Government program. 


\section{References}

Brand S, Solanki B, Foster DB, Czarnota GJ, Kolios MC. Monitoring of cell death in epithelial cells using high frequency ultrasound spectroscopy. Ultrasound Med Biol 2009;35:482-493

Chen X, Phillips D, Schwarz KQ, Mottley JG, and Parker KJ. The measurement of backscatter coefficient from a broadband pulse-echo system: a new formulation. IEEE Trans Ultras Ferroelectr Freq Control $1997 ; 44: 515525$

Czarnota GJ, Kolios MC, Vaziri H, Benchimol S, Ottensmeyer FP, Sherar MD, Hunt JW. Ultrasonic biomicroscopy of viable, dead and apoptotic cells. Ultrasound Med Biol 1997; 23:961-965

Falou O, Rui M, Kaffas AE, Kumaradas JC, and Kolios MC. The measurement of ultrasound scattering from individual micron-sized objects and its application in single cell scattering. J Acoust Soc Am 2010; 128:894-902

Feleppa EJ, Liu T, Kalisz A, Shao MC, Fleshner N, Reuter V. Ultrasonic spectral-parameter imaging of the prostate. Int. J. Imag. Syst. Technol. $1997 ; 8: 11-25$.

Franceschini E, Guillermin R. Experimental assessment of four ultrasound scattering models for characterizing concentrated tissue-mimicking phantoms. J Acoust Soc Amer 2012; 132:3735-3747.

Franceschini E, Guillermin R, Tourniaire F, Roffino S, Lamy E, Landrier JF. Structure Factor Model for understanding the measured backscatter coefficients from concentrated cell pellet biophantoms. J Acoust Soc Amer $2014 ; 135: 3620-3631$. 
Franceschini E, de Monchy R, Mamou J. Quantitative characterization of tissue microstructure in concentrated cell pellet biophantoms based on the structure factor model. IEEE Trans Ultras Ferroelectr Freq Control 2016; 63:1321-1334.

Griffith WL, Triolo R, Compere AL. Analytical scattering function of a polydisperse Percus-Yevick fluid with Schulz distributed diameters. Phys Rev A 1987; 35:2200-2206.

Han A, Abuhabsah R, Blue JP, Sarwate S, OBrien WD. The measurement of ultrasound backscattering from cell pellet biophantoms and tumors ex vivo. J Acoust Soc Am 2013; 134:686693.

Han A, O’Brien WD. Structure function for high-concentration biophantoms of polydiperse scatterer sizes. IEEE Trans Ultras Ferroelectr Freq Control 2015; 62:303-318.

Hunt J.W., Worthington A.E., Xuan A., Kolios M.C., Czarnota G.J. and Sherar M.D., A model based upon pseudo regular spacing of cells combined with the randomisation of the nuclei can explain the significant changes in high-frequency ultrasound signals during apoptosis. Ultrasound Med Biol 28, 2002, 217226.

Insana MF, Wagner RF, Brown DG, Hall TJ. Describing small-scale structure in random media using pulse-echo ultrasound. J Acoust Soc Am $1990 ; 87: 179-192$

Kolios MC, Czarnota GJ, Lee M, Hunt JW, Sherar MD. Ultrasonic spectral parameter characterization of apoptosis. Ultrasound Med Biol 2002; 28:589-597

Lavarello RJ, Ridgway WR, Sarwate SS, Oelze ML. Characterization 
of thyroid cancer in mouse models using high-frequency quantitative ultrasound techniques. Ultrasound Med Biol 2013; 39:2333-2341.

Lizzi FL, Ostromogilsky M, Feleppa EJ, Rorke MC, Yaremko MM. Relationship of ultrasonic spectral parameters to features of tissue microstructure. IEEE Trans Ultrason Ferroelect Freq Contr. 1986; 33:319-329 Mamou J, Coron A, Hata M, Machi J, Yanagihara E, Laugier P, Feleppa E. Three-dimensional high-frequency characterization of cancerous lymph nodes. Ultrasound Med Biol 2010; 36:361-375.

Muleki-Seya P, Guillermin R, Guglielmi J, Chen J, Pourcher T, Konofagou E, Franceschini E. High frequency quantitative ultrasound spectroscopy of excised canine livers and mouse tumors using the structure factor model. IEEE Trans Ultras Ferroelectr Freq Control 2016; 63:1335-1350.

Oelze ML, O'Brien WD, Blue JP, Zachary JF. Differentiation and characterization of rat mammary fibroadenomas and 4T1 mouse carcinomas using quantitative ultrasound imaging. IEEE Trans. Med. Imaging 2004; 23:764-771.

Oelze ML, O'Brien WD. Application of three scattering models to characterization of solid tumors in mice. Ultrasonic Imaging 2006; 28:83-96. Pasternak MM, Sadeghi-Naini A, Ranieri SM, Giles A, Oelze ML, Kolios MC, Czarnota GJ, High-frequency ultrasound detection of cell death: spectral differentiation of different forms of cell death in vitro. Oncoscience 2016; 3:275-287.

Qiao L, Koutsos M, Tsai LL, Kozoni V, Guzman J, Shiff SJ, Rigas B. Staurosporine inhibits the proliferation, alters the cell cycle distribution and induces apoptosis in HT-29 human colon adenocarcinoma cells. 
Cancer Letters 1996; 107:83-89.

Sannachi L, Tadayyon H, Sadeghi-Naini A, Tran W, Sonal G, Wright F, Oelze M, Czarnota GJ. Non-invasive evaluation of breast cancer response to chemotherapy using quantitative backscatter parameters. Medical images analysis 2015; 20:224-236.

Strohm EM, Czarnota GJ, Kolios MC. Quantitative measurements of apoptotic cell properties using acoustic microscopy. IEEE Trans Ultras Ferroelectr Freq Control 2010; 57:2293-2304.

Tadayyon H, Sannachi L, Sadeghi-Naini A, Al-Mahrouki A, Tran WT, Kolios MC, Czarnota GJ. Quantification of ultrasonic properties of in vivo tumor cell death in mouse models of breast cancer. Translational Oncology 2015; 8:463-473.

Tadayyon H, Sannachi L, Gangeh MJ, Kim C, Ghandi S, Trudeau M, Pritchard K, Tran WT, Slodkowska E, Sadeghi-Naini A, Czarnota GJ. Chemotherapy response and survival in breast cancer patients using quantitative ultrasound, Scientific reports 2017; 7:45733.

Taggart LR, Baddour RE, Giles A, Czarnota GJ, Kolios MC. Ultrasonic characterization of whole cells and isolated nuclei. Ultrasound Med Biol 2007; 33:389-401.

Vlad RM, Alajez NM, Giles A, Kolios MC, Czarnota GJ. Quantitative ultrasound characterization of cancer radiotherapy effects in vitro. Int $\mathrm{J}$ Radiat Oncol Biol Phys 2008;72:12361243.

Vlad RM, Saha RK, Alajez NM, Ranieari S, Czarnota GJ, Kolios MC. An increase in cellular size variance contributes to the increase in ultrasound backscatter during cell death. Ultrasound Med Biology 2010; 
$596 \quad 36: 1546-1558$.

${ }_{597}$ Weiss EC, Anastasiadis P, Pilarczyk G, Lemor RM, Zinin PV. Mechanical 598 properties of single cells by high-frequency time-resolved acoustic microscopy. IEEE Trans Ultras Ferroelectr Freq Control 2007; 54:2257-2271. Yao LX, Zagzebski JA, Madsen EL. Backscatter coefficient measurements using a reference phantom to extract depth-dependent instrumentation factors. Ultrasonic Imaging 1990; 12:58-70. 


\section{Tables}

${ }_{605}$ Table 1: Mean radius $a_{s}$ (in $\left.\mu \mathrm{m}\right)$ and gamma width factor $\zeta_{s}$ for small cellular fragments. Mean radius $a_{l}$ and gamma width factors $\zeta_{l}$ for

607 large cells, and corresponding percentage of volume fraction occupied by large cells $\Phi_{l}$. Results are presented for the four series of experiments (i.e. ST dose and time effect) and for the different conditions (dose effect $C_{i=1 \ldots 6}=0,0.125,0.25,0.50,0.75$ and $1 \mu \mathrm{M}$ and time effect

611 $C_{i=1 \ldots 6}=0,6,12,24,36$ and 48 hours ). 


\begin{tabular}{|c|c|c|c|c|c|}
\hline & & Dose 1 & Dose 2 & Dose 3 & Time \\
\hline \multirow{3}{*}{$C_{1}$} & $a_{s}, \zeta_{s}$ &,-- & $2.5,15.2$ & $2.4,9.3$ & $2.5,15.1$ \\
\hline & $a_{l}, \zeta_{l}$ & $6.3,53.6$ & $6.4,42$ & $6.8,55.6$ & $6.4,95$ \\
\hline & $\Phi_{l}$ & 1 & 1 & 0.99 & 0.99 \\
\hline \multirow{3}{*}{$C_{2}$} & $a_{s}, \zeta_{s}$ & $2.7,5.1$ & $2.6,11.6$ & $2.3,2.3$ & $2.3,8.8$ \\
\hline & $a_{l}, \zeta_{l}$ & $7.3,23.9$ & $5.5,13.7$ & $7.3,73.1$ & $6.7,50.2$ \\
\hline & $\Phi_{l}$ & 0.91 & 0.96 & 0.93 & 0.97 \\
\hline \multirow{3}{*}{$C_{3}$} & $a_{s}, \zeta_{s}$ & $2.7,5.8$ & $2.6,13.5$ & $2.7,5.4$ & $2.6,6.5$ \\
\hline & $a_{l}, \zeta_{l}$ & $7.2,16.4$ & $5.6,11.7$ & $7.5,57.9$ & $6.9,50.4$ \\
\hline & $\Phi_{l}$ & 0.92 & 0.97 & 0.93 & 0.93 \\
\hline \multirow{3}{*}{$C_{4}$} & $a_{s}, \zeta_{s}$ & $2.6,7.7$ & $3.7,4.1$ & $2.3,2.3$ & $2.3,3.9$ \\
\hline & $a_{l}, \zeta_{l}$ & $5.3,10.1$ & $7.1,36.2$ & $7.2,58.3$ & $7.1,55.3$ \\
\hline & $\Phi_{l}$ & 0.86 & 0.81 & 0.85 & 0.74 \\
\hline \multirow{3}{*}{$C_{5}$} & $a_{s}, \zeta_{s}$ & $2.6,10.1$ & $2.4,13.8$ & $2.4,3.9$ & $2.4,5.0$ \\
\hline & $a_{l}, \zeta_{l}$ & $5.5,19.4$ & $4.8,18.3$ & $6.9,55.2$ & $7.6,48.8$ \\
\hline & $\Phi_{l}$ & 0.84 & 0.92 & 0.80 & 0.75 \\
\hline \multirow{3}{*}{$C_{6}$} & $a_{s}, \zeta_{s}$ & $2.4,12.4$ & $2.5,10.3$ & $2.4,5.5$ & $2.0,4.0$ \\
\hline & $a_{l}, \zeta_{l}$ & $5.7,10.6$ & $5.2,22.7$ & $6.5,39.4$ & $8.5,29$ \\
\hline & $\Phi_{l}$ & 0.93 & 0.88 & 0.74 & 0.80 \\
\hline
\end{tabular}


617

618

Table 2: Examples of QUS parameters (ASD* and $\sigma_{D}^{*}$ in $\mu \mathrm{m}$ and $\mathrm{AAC}^{*}$ in $\mathrm{dB} . \mathrm{cm}^{-3}$ ) estimated by the polydisperse SFM for the time effect experiment. The novel approach was tested by considering two different contraint conditions: $0 \leq \phi \leq 1$ or $0.68 \leq \phi \leq 1$.

\begin{tabular}{|c|c|c|c|c|}
\hline & & Expected & QUS with & QUS with \\
parameters & $\begin{array}{c}\text { constraint } \\
0 \leq \phi \leq 1\end{array}$ & $\begin{array}{c}\text { constraint } \\
0.68 \leq \phi \leq 1\end{array}$ \\
\hline $6 \mathrm{~h}$ & $\begin{array}{c}\mathrm{ASD}^{*}, \sigma_{D}^{*} \\
\mathrm{AAC}^{*}\end{array}$ & $\begin{array}{c}13.4,1.86 \\
64.3\end{array}$ & $\begin{array}{c}4.7,0.57 \\
70.9\end{array}$ & $\begin{array}{c}11.7,1.17 \\
63.3\end{array}$ \\
\hline \multirow{2}{*}{$24 \mathrm{~h}$} & $\mathrm{ASD}^{*}, \sigma_{D}^{*}$ & $13.8,1.91$ & $9.0,2.36$ & $12.2,1.33$ \\
& $\mathrm{ASD}^{*}, \sigma_{D}^{*}$ & $14.2,1.89$ & $7.9,2.04$ & $11.3,1.31$ \\
& $\mathrm{AAC}^{*}$ & 63.1 & 58.5 & 66.1 \\
\hline $36 \mathrm{~h}$ & $\mathrm{ASD}^{*}, \sigma_{D}^{*}$ & $15.2,2.15$ & $4.6,1.26$ & $11.6,1.53$ \\
& $\mathrm{AAC}^{*}$ & 62.7 & 73.6 & 67.0 \\
\hline $48 \mathrm{~h}$ & $\mathrm{ASD}^{*}, \sigma_{D}^{*}$ & $17.0,3.10$ & $4.5,1.63$ & $15.3,2.30$ \\
$\mathrm{AAC}^{*}$ & 63.3 & 83.7 & 66.9 \\
\hline
\end{tabular}




\section{Figure Captions}

Figure 1: Histological images of HT29 cell pellet biophantoms (a) non treated and (b) treated with staurosporine $1 \mu \mathrm{M}$.

Figure 2: a) Typical examples of cell radius distribution. The solid lines are the direct measurements using the Scepter cell counter and the dashed lines correspond to the fitting curves with the cell radius probability density function $F(x)$ given by Eq. (6). (b) Histograms of the cell volume distribution $V(x)=F(x)(4 / 3) \pi x^{3}$. The histograms are normalized to one by dividing each count from the cell volume distribution to the maximum count.

Figure 3: a) Ultrasonic probe and cell pellet biophantom in a well immersed in PBS. b) and c) Examples of B-mode images of non-treated and treated cell pellet biophantoms obtained with the $20-\mathrm{MHz}$ center frequency probe. The treated cell sample corresponds to the sample treated with staurosporine $(\mathrm{ST})$ at $0.5 \mu \mathrm{M}$ for 36 hours.

Figure 4: Comparison between measured $B S C_{\text {meas }}$ (solid lines) and theoretical $B S C_{\text {theo }}$ predicted by the polydisperse structure factor model (dashed lines) for the dose effect 1 (a) and b) and for the time effect (c) and (d).

Figure 5: Comparison of mean $B S C_{\text {theo }}$ in the 10-32 MHz bandwidth predicted by the polydisperse structure factor model with the mean $B S C_{\text {meas }}$ in the 10-32 MHz bandwidth. 
641 Figure 6: (a) Examples of $\mathrm{ASD}^{*}$ and $\mathrm{AAC}^{*}$ estimated by the fluid sphere model for the series of experiments dose effect 1. For each studied dose, five symbols are represented and correspond to the five acquired B-mode images. (b) Spectral intercept as a function of percentage of dead cells for the four experimental series. (c) Standard deviation of the scatterer diameter distribution $\sigma_{D}^{*}$ estimated by the polydisperse SFM as a function of the percentage of dead cells for the four experimental series. 


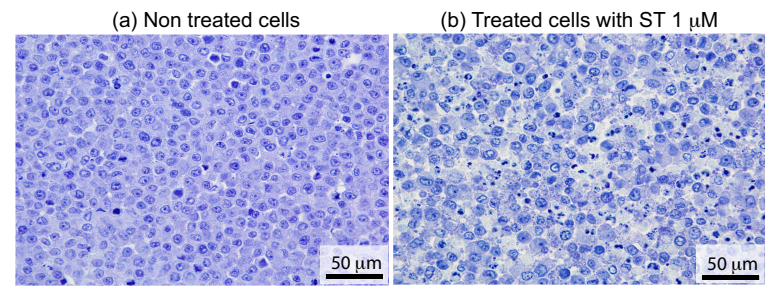

Figure 1: Histological images of HT29 cell pellet biophantoms (a) non treated and (b) treated with staurosporine $1 \mu \mathrm{M}$. 

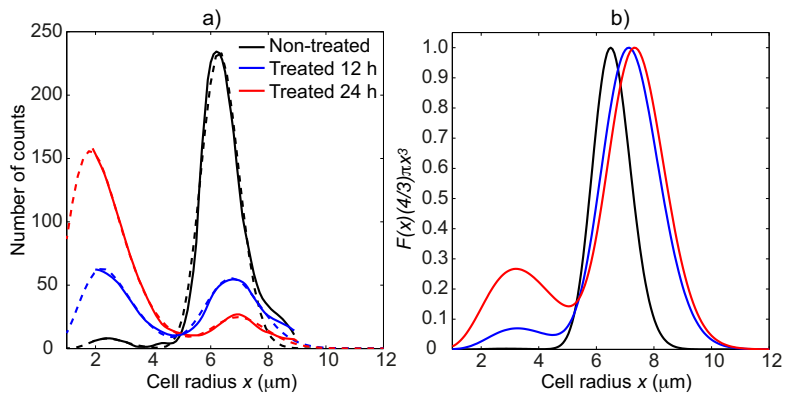

Figure 2: (a) Typical examples of cell radius distribution. The solid lines are the direct measurements using the Scepter cell counter and the dashed lines correspond to the fitting curves with the cell radius probability density function $F(x)$ given by Eq. (6). (b) Histograms of the cell volume distribution $V(x)=F(x)(4 / 3) \pi x^{3}$. The histograms are normalized to one by dividing each count from the cell volume distribution to the maximum count. 

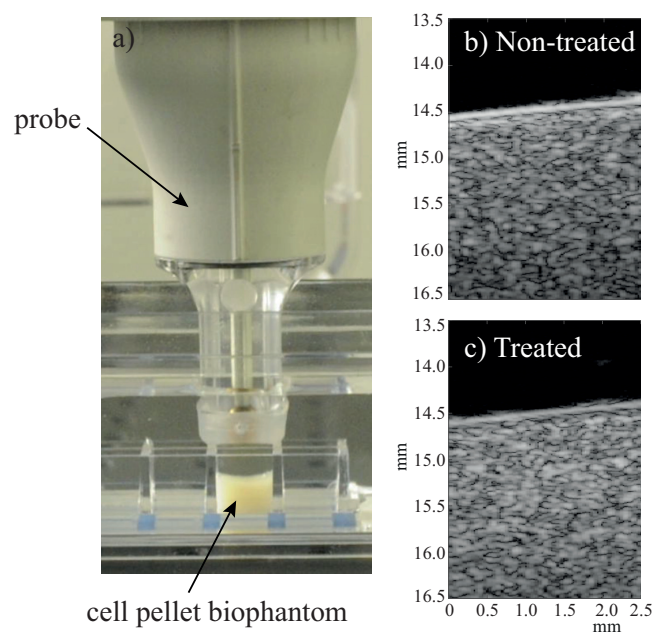

Figure 3: a) Ultrasonic probe and cell pellet biophantom in a well immersed in PBS. b) and c) Examples of B-mode images of non-treated and treated cell pellet biophantoms obtained with the $20-\mathrm{MHz}$ center frequency probe. The treated cell sample corresponds to the sample treated with staurosporine (ST) at $0.5 \mu \mathrm{M}$ for 36 hours. 


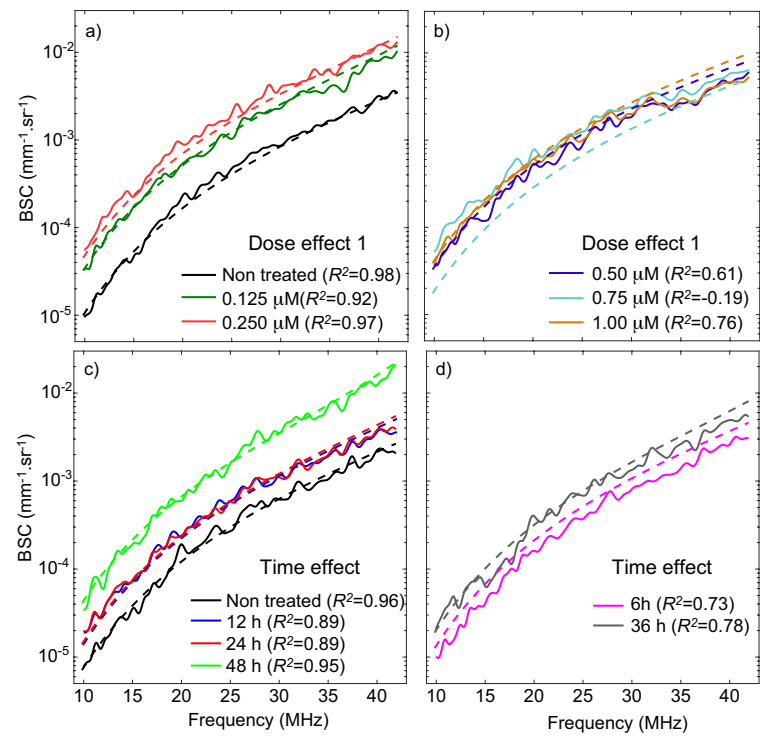

Figure 4: Comparison between measured $B S C_{\text {meas }}$ (solid lines) and theoretical $B S C_{\text {theo }}$ predicted by the polydisperse structure factor model (dashed lines) for the dose effect 1 (a) and b) and for the time effect (c) and (d). 


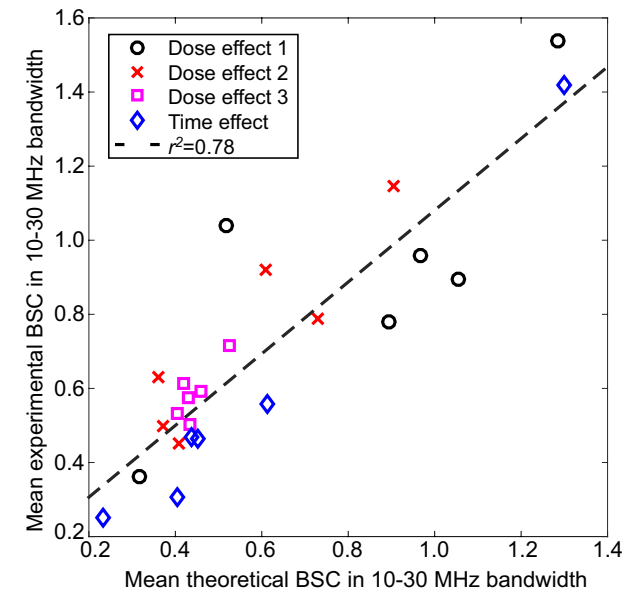

Figure 5: Comparison of mean $B S C_{\text {theo }}$ in the $10-32 \mathrm{MHz}$ bandwidth predicted by the polydisperse structure factor model with the mean $B S C_{\text {meas }}$ in the $10-32 \mathrm{MHz}$ bandwidth. 

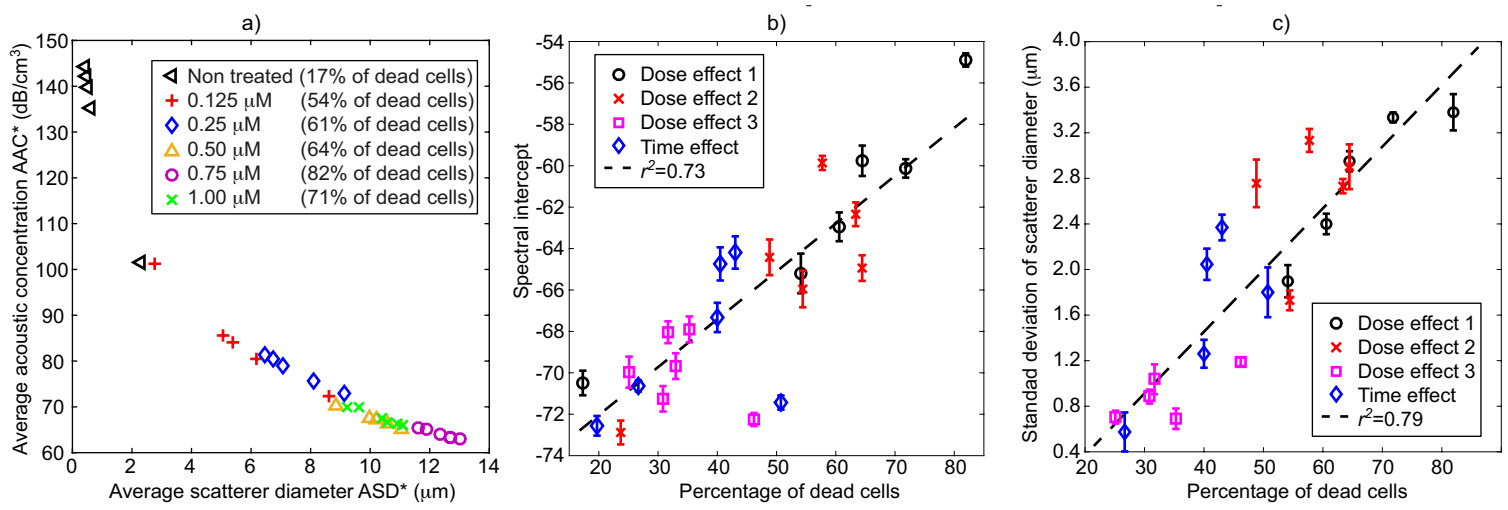

Figure 6: (a) Examples of $\mathrm{ASD}^{*}$ and $\mathrm{AAC}^{*}$ estimated by the fluid sphere model for the series of experiments dose effect 1. For each studied dose, five symbols are represented and correspond to the five acquired B-mode images. (b) Spectral intercept as a function of percentage of dead cells for the four experimental series. (c) Standard deviation of the scatterer diameter distribution $\sigma_{D}^{*}$ estimated by the polydisperse SFM as a function of the percentage of dead cells for the four experimental series. 